\title{
The response in the blood of piglets to oral doses of galactose and glucose and intravenous administration of galactose
}

\author{
BY P. H. BIRD AND P. E. HARTMANN \\ Department of Biochemistry, The University of Western Australia, Nedlands, WA 6009, Australia
}

(Received 29 October 1992 - Revised 30 April 1993 - Accepted 8 June 1993)

\begin{abstract}
The kinetics of the response in the blood of piglets to physiological oral intakes of galactose and glucose, and intravenous administration of galactose are described. Following the intravenous administration of galactose to 2- and 10-d-old piglets ( $n$ 7), the half-life was 7.98 (SD 0.75) and 7.99 (SD 1.89) min respectively, and efficient elimination rate was 9.09 (SD 2.15) and 8.75 (SD 0.79) \% per min respectively. The turnover of galactose in the piglets was $100.3 \mu \mathrm{g} / \mathrm{min}$ per $\mathrm{kg}$ body weight. These observations demonstrate that galactose was rapidly removed from the blood of the piglets. While the dosing and sampling procedures stimulated hyperglycaemia, they had no effect on the concentration of galactose in the peripheral plasma. The galactose area under the curve (adjusted to the plasma volume of the animal) following a dose of either galactose or galactose plus glucose was 1.75 (SD 0.15) and 1.95 (SD 0.14) arbitrary units respectively in 2-d-old piglets and 1.96 (SD 0.26) and 1.98 (SD 0.10) arbitrary units respectively in 10-d-old piglets. Since the presence of glucose did not lower the adjusted area under the curve for galactose in the peripheral blood, the effect of glucose on the metabolism of galactose in piglets was more like that reported for rats than that for man, guinea-pigs or mice. It is suggested that the galactose moiety of lactose may make an important contribution to the replenishment of liver glycogen in the neonatal piglet.
\end{abstract}

Galactose: Glucose: Blood galactose kinetics: Piglet

The oral administration of galactose and/or glucose results in different temporal changes in the concentration of galactose and glucose in the blood (Newstead, 1979; Williams et al. 1983; Williams \& Owens, 1984). For example, Williams et al. (1983) demonstrated in man that the increase in the concentration of galactose in the peripheral blood following an oral dose of galactose was depressed by the inclusion of glucose in the dose. Furthermore, this effect of glucose did not appear to be directly related to the digestion and absorption processes, as the intravenous administration of glucose had a similar effect on the concentration of galactose in the blood following an oral dose of galactose.

Investigation into the elimination of galactose from the peripheral blood after physiological intakes has been hindered by assay sensitivity. However, recently Kaempf $e t$ al. (1990) used sensitive methods to describe the kinetics of galactose in the peripheral blood of lambs after natural suckings. Furthermore, the development of a sensitive bioluminescence assay by Arthur et al. (1989) enabled the determination of the changes in the concentration of galactose in the blood of piglets after natural suckings (Holmes et al. 1990). This latter study demonstrated that whereas the concentration of glucose in the blood of piglets ranged from 5.7 (SD 0.3) $\mathrm{mm}$ to 7.7 (SD 0.3) $\mathrm{mm}$, the concentration of galactose ranged from 19 (SD 2) $\mu \mathrm{M}$ to 122 (SD 21) $\mu \mathrm{M}$. However, the interaction between the administration of glucose and galactose on the kinetics of the elimination of galactose from the blood of piglets has not been reported. Therefore, we investigated the influence of physiological oral doses of galactose and/or glucose on the concentrations of these 
monosaccharides in the peripheral blood of 2- and 10-d-old piglets. We have also investigated the kinetics and calculated the elimination rate of galactose from the blood of piglets following the intravenous injection of galactose.

\section{MATERIALS AND METHODS}

\section{Animals}

Healthy 2- and 10-d-old piglets ( $n$ 74) with mean body weights of 1.8 (SD 0.3 ) and 3.5 (SD $0.4) \mathrm{kg}$ respectively were studied. Groups of test animals (up to seven piglets) were compiled from different litters where up to two piglets were selected at random from any one litter. The piglets were separated from their sow by partitioning the farrowing crate.

\section{Intravenous doses}

Piglets (2-d-old, $n 7 ; 10$-d-old, $n 7$ ) were prevented from sucking for at least $1 \mathrm{~h}$ and then administered an intravenous dose of galactose in water $(250 \mathrm{~g} / \mathrm{l} ; 60 \mathrm{mg}$ galactose $/ \mathrm{kg}$ body weight) by a single injection into an ear vein. The administration time was between 10 and $30 \mathrm{~s}$. Zero time was taken as the time when half the volume of the solution of galactose had been administered. The veins in the opposite ear of the piglet were pricked and blood samples $(60 \mu \mathrm{l})$ were collected at 5-10 min intervals for $35 \mathrm{~min}$ after the dose.

\section{Oral doses}

Groups of 2- and 10-d-old piglets were prevented from sucking for at least $1 \mathrm{~h}$ and then the piglets within each age-group were assigned at random to one of five treatment groups. A blood sample was then collected by pricking the piglet's ear vein and, depending on the group, each piglet underwent one of the following treatments: (a) ND, no oral dose (2-dold, $n 6 ; 10$-d-old, $n 6$ ); (b) $\mathrm{H}_{2} \mathrm{O}$, an oral dose of deionized water (15 ml; 2-d-old, $n 6 ; 10$ d-old, $n 6)$; (c) GLU, an oral dose of deionized water $(15 \mathrm{ml})$ containing 0.675 g glucose $(2-$ d-old, $n 7 ; 10$-d-old, $n 7$ ); (d) GAL, an oral dose of deionized water (15 ml) containing 0.675 g galactose (2-d-old, $n 6 ; 10$-d-old, $n 6) ;(\mathrm{e}) \mathrm{GAL}+\mathrm{GLU}$, an oral dose of deionized water $(15 \mathrm{ml}$ ) containing 0.675 g galactose plus 0.675 g glucose (2-d-old, $n 5 ; 10$-d-old, $n 5$ ).

The administration time of the dose was between 0.5 and 2 min and zero time was taken as the time when half the volume of the solution had been administered. The ear veins of the piglets were pricked and blood samples $(60 \mu \mathrm{l})$ were collected at $3-10 \mathrm{~min}$ intervals for $40 \mathrm{~min}$ and then at $60 \mathrm{~min}$ after the dose. The blood plasma was separated by centrifugation and stored at $-20^{\circ}$ for analysis.

\section{Biochemical analysis}

The plasma $(20 \mu \mathrm{l})$ was deproteinized with $200 \mu \mathrm{l} 0.6 \mathrm{M}$-perchloric acid (Arthur et al. 1989). The concentration of galactose was determined by the bioluminescence method of Arthur et al. (1989) and the concentration of glucose was measured by the glucose oxidase (EC 1.1.3.4) method of Bergmeyer \& Bern't (1974) as modified by Holmes et al. (1990).

\section{Kinetic analysis}

After administration of either an intravenous or an oral dose of galactose, the concentration of galactose in the plasma $v$. time was plotted. The elimination phase of the curve for each piglet was determined with a least squares linear regression line, based on the concentrations of galactose in the peripheral plasma (between the peak and $35 \mathrm{~min}$ after injection for intravenous doses, and between the peak and $60 \mathrm{~min}$ after the administration of the oral doses) whose predicted values were within a $95 \%$ confidence band of the fitted values. The half-life was determined from the elimination slope given that $C=C_{0} \cdot e^{-k t}$ (Tygstrup \& Winkler, 1954) where $C$ is the concentration of galactose, $C_{o}$ is the concentration of 
galactose at time $0, e$ is the base of natural logarithms, $k$ is the elimination rate constant (slope) and $t$ is time. All calculations were carried out using StatView, SE + Graphics ${ }^{(\mathbb{M N}}$ II, v 1.03 (Abacus Concepts Inc., Berkeley, CA, USA).

Following oral dosing the concentrations of galactose in the blood $v$. time were plotted and the area under the curve (AUC) for each piglet was calculated by the trapezoid method reported by Williams et al. (1983), which was derived from Yeh \& Kwan (1978). Previous reports (Barber et al. 1955) and preliminary studies in our laboratory have demonstrated that piglets up to 3 weeks of age suck similar amounts of milk. Thus, in our study it was appropriate to keep the oral dose of carbohydrate constant for piglets of different ages. Since piglets of different body weights were administered the same amount of either glucose or galactose, the heavier piglets distributed the absorbed monosaccharide in a larger volume of plasma. Therefore, it was necessary to adjust the AUC to account for the different dilution of the monosaccharides in piglets of different weights.

To allow comparisons to be made between piglets of different body weights, the AUC for individual piglets were standardized as follows: (1) an estimate of the total blood volume was based on the formulas of Engelhardt (1966) and Mount \& Ingram (1971). The formula of Mount \& Ingram (1971) was modified and checked against data of both Ramirez et al. (1963) and Engelhardt (1966). Thus, based on Engelhardt's (1966) formulas and the plot of the logarithm of blood volume $(\mathrm{ml} / 100 \mathrm{~g}$ body weight $) v$. the logarithm of body weight $(\mathrm{kg})$, the modified formula used was: blood volume $(\mathrm{ml})=$ weight $(\mathrm{kg})^{0.932} \times 95$, where 95 is the blood volume ( $\mathrm{ml})$ of a $1 \mathrm{~kg}$ piglet; (2) the plasma volume was estimated for individual piglets using the relationship between blood volume, plasma volume and body weight of piglets derived by Ramirez et al. (1963); (3) the plasma volume of the piglet was expressed relative to an arbitrary reference plasma volume $(600 \mathrm{ml})$. Thus, for each piglet plasma volume: reference volume could be calculated to allow comparisons between piglets of different body weights; (4) this ratio was used to adjust the galactose AUC of each piglet to provide an index of the amount of galactose that was present in the peripheral circulation. This index was termed the 'adjusted galactose AUC'. For example, the adjusted AUC for a piglet weighing $3 \mathrm{~kg}$ was determined as follows: total blood volume: weight $(\mathrm{kg})^{0.932} \times 95=265 \mathrm{ml}$; plasma volume: $265 \times 0.7=185 \mathrm{ml}$; an adjustment ratio was then obtained: $600 / 185=3 \cdot 2$; assuming the galactose AUC for this piglet was 5000 arbitrary units, then the adjusted galactose AUC was $5000 / 3 \cdot 2=1563$ arbitrary units.

Following an oral dose the concentrations of glucose in the blood $v$. time were plotted and the increase in the concentration of glucose was calculated from the difference between the concentration of glucose before the treatment and the peak concentration of glucose during the treatment for each piglet.

\section{Statistical analysis}

Significant differences between the half-lives of galactose were determined by unpaired Student's $t$ test. Significant differences between either the adjusted galactose AUC or the increase in the concentration of glucose following the different treatments were determined by two-factor analysis of variance using StatView, SE + Graphics II, v 1.03 (Abacus Concepts Inc.). The results are expressed as means with either standard errors of the means (SE) or standard errors of the difference between means (SED).

\section{RESULTS}

Intravenous doses of galactose

The changes in the concentrations of galactose in the plasma of 2- and 10-d-old piglets following the intravenous dose of galactose are illustrated in Fig. 1. The half-life for the elimination of galactose from the plasma was 7.98 (SE 0.28) min for the 2-d-old piglets $(n 7)$, 


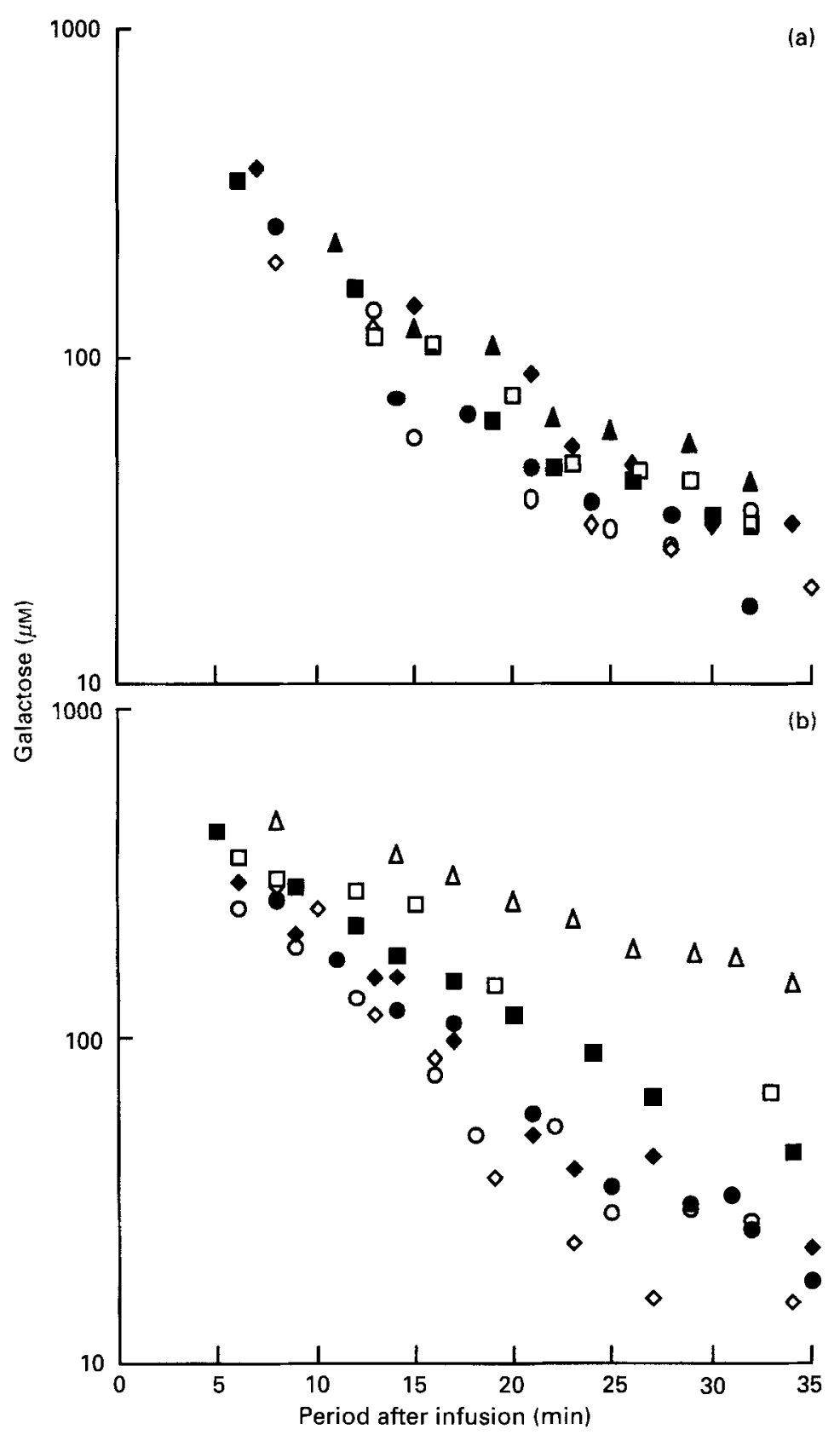

Fig. 1 The concentration of galactose in the plasma (logarithm scale) with time in (a) 2-d-old piglets and (b) 10 d-old piglets $(n)$ following an intravenous dose of galactose $(60 \mathrm{mg} / \mathrm{kg}$ body weight $)$. Values shown by different symbols are those for individual pigs. For details of treatments and procedures, see pp. 554-555.

which was not significantly different $(P=0.042)$ from the half-life of galactose in the 10 d-old piglets $(9 \cdot 10(\mathrm{SE} 1 \cdot 29) \mathrm{min} ; n 7)$. The regression equations for the mean concentration of galactose in the plasma $v$. time for the 2- and 10-d-old piglets (not including piglet 3 ) were: $\log y=2.64-0.39 x,(r 0.94)$ and $\log y=2.69-0.42 x,(r 0.92)$ respectively. The 


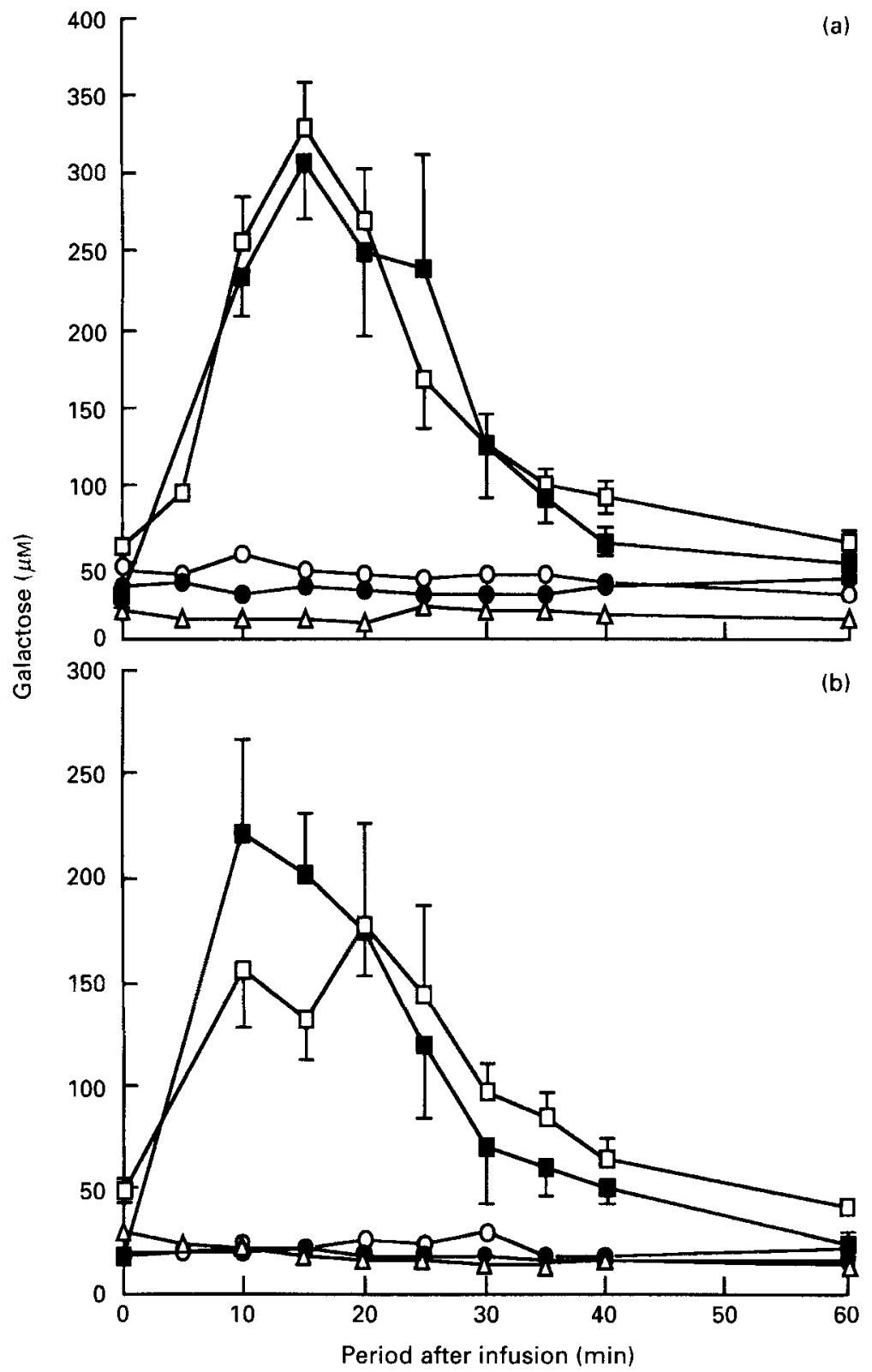

Fig. 2. The concentrations of galactose in the plasma with time in (a) 2-d-old piglets and (b) 10-d-old piglets following no previous administration of an oral dose of any solution (ND treatment; $\triangle ; n 6)$; an oral dose of deionized water $(15 \mathrm{ml})\left(\mathrm{H}_{2} \mathrm{O}\right.$ treatment; $\left.; n 6\right)$; an oral dose of glucose $(0.675 \mathrm{~g})(\mathrm{GLU}$ treatment; $\bigcirc ; n)$; an oral dose of galactose $(0.675 \mathrm{~g}$ ) (GAL treatment; $\square ; n 6)$; an oral dose of galactose $(0.675 \mathrm{~g})$ plus glucose $(0.675 \mathrm{~g})(\mathrm{GAL}+\mathrm{GLU}$ treatment; $\square ; n 5)$. The values are presented as means with their standard errors represented by vertical bars. For details of treatments and procedures, see pp. 554-555. 
Table 1. The mean adjusted plasma galactose area under the curve (AUC; arbitrary units) and the mean increase in the concentration of glucose $(\mathrm{mM})$ in 2-and 10-d-old piglets following either no dose $(N D)$ or oral treatments of water $\left(\mathrm{H}_{2} \mathrm{O}\right)$, glucose $(G L U)$, galactose $(G A L)$ and galactose plus glucose $(G A L+G L U)^{*}$

(Means with their standard error of the difference between means)

\begin{tabular}{|c|c|c|c|c|c|c|}
\hline Age (d) & No dose & $\mathrm{H}_{2} \mathrm{O}$ & GLU & GAL & $\begin{array}{c}\text { GAL+ } \\
\text { GLU }\end{array}$ & SED \\
\hline \multicolumn{7}{|l|}{ Galactose } \\
\hline 2 & $0 \cdot 16^{\mathrm{a}}$ & $0 \cdot 40^{\mathrm{ab}}$ & $0.54^{i j}$ & $1 \cdot 75^{\mathrm{c}}$ & $1.95^{\circ}$ & $0 \cdot 16$ \\
\hline 10 & $0 \cdot 40^{\mathrm{a}}$ & $0 \cdot 38^{\mathrm{a}}$ & $0.52^{\mathrm{a}}$ & $1 \cdot 85^{b}$ & $1.98^{b}$ & $0 \cdot 16$ \\
\hline \multicolumn{7}{|l|}{ Glucose } \\
\hline 2 & $0.56^{\mathrm{a}}$ & $0 \cdot 93^{\mathrm{a}}$ & $3.08^{b}$ & $1 \cdot 81^{\mathrm{c}}$ & $2 \cdot 57^{\mathrm{bc}}$ & 0.45 \\
\hline 10 & $0.62^{\mathrm{a}}$ & $1.56^{\mathrm{b}}$ & $2.61^{c}$ & $1.55^{\mathrm{b}}$ & $2 \cdot 37^{\mathrm{bcd}}$ & 0.45 \\
\hline
\end{tabular}

$\mathbf{a}, \mathbf{b}, c, \mathrm{~d}$ Mean values in horizontal rows with unlike superscripts were significantly different (two-way ANOVA); $P<0.05$.

* For details of treatments and procedures, see pp. 554-556.

regression equations for the concentration of galactose in the plasma $v$. time for the 2 - and 10-d-old piglets were: $\log y=2.64-0.039 x,(r 0.94)$ and $\log y=2.64-0.033 x,(r 0.76)$ respectively.

\section{Oral doses of sugars and plasma galactose}

The half-life of galactose in the plasma of the piglets given an oral dose of galactose was $9 \cdot 29$ (SE 1.21) min with a confidence interval of 5.60-12.97 min $(n 6)$ and 7.15 (SE 0.92) min with a confidence interval of 4.36-9.94 $\min (n 6)$ for the 2- and 10-d-old piglets respectively. There was no significant difference between these values and the results for the half-lives for galactose in the plasma of the 2- (confidence interval of 7.92-8.68 min) and 10-d-old (confidence interval of 5.94-12.26 min) piglets given an intravenous dose of galactose.

The concentrations of galactose in the plasma of the 2- and 10-d-old piglets after the different treatments are illustrated in Fig. 2. The adjusted galactose AUC for the 2- and 10$\mathrm{d}$-old piglets after the different treatments are shown in Table 1. There was no significant increase in the concentration of galactose in the blood after the $\mathrm{ND}, \mathrm{H}_{2} \mathrm{O}$ and GLU treatments in either the 2- or 10-d-old piglets. However, there was a marked increase in the concentration of galactose (Fig. 2) resulting in a significantly higher $(P<0.0001)$ adjusted galactose AUC after oral doses of either galactose (GAL treatment) or galactose plus glucose (GAL + GLU treatment), compared with the $\mathrm{ND}, \mathrm{H}_{2} \mathrm{O}$ and GLU treatments in both the 2- and 10-d-old piglets (Table 1). There was no significant difference in the adjusted galactose AUC following the GAL treatment compared with the GAL + GLU treatment.

\section{Oral doses of sugars and plasma glucose}

The increases in the concentrations of glucose between the pretreatment blood sample and the peak value of glucose after oral dosing are shown in Table 1 . The mild stress associated with all the treatments resulted in an increase in the concentration of plasma glucose in the piglets. The increase in the concentration of glucose in the plasma of the 10-d-old piglets following the $\mathrm{H}_{2} \mathrm{O}$ treatment was significantly greater $(P<0.05)$ than the increase following the ND treatment. However, this increase was not observed in the 2-d-old piglets.

The increase in the concentration of glucose in the plasma of both 2- and 10-d-old piglets 
was significantly higher following the GLU ( $P<0.0001$ for both ages), GAL $(P<0.01$ and $0 \cdot 05$, respectively) and GAL + GLU $(P<0.001$ for both ages $)$ treatments compared with the increase following the ND treatment. Although the change in plasma glucose after the GLU treatment was significantly higher than that after the $\mathrm{H}_{2} \mathrm{O}$ treatment in both 2- $(P$ $<0.0001)$ and 10 -d-old $(P<0.05)$ piglets, there was no difference in the increase of glucose between the $\mathrm{H}_{2} \mathrm{O}$ treatment and the GAL $(P>0.9)$ and GAL $+\mathrm{GLU}(P>0.05)$ treatments in the 10-d-olds and between the $\mathrm{H}_{2} \mathrm{O}$ treatment and the GAL $(P>0.05)$ treatment in the 2-d-olds. Furthermore, the change in the plasma concentration of glucose following the GLU treatment was not significantly different from the change following the GAL + GLU treatment in both the $2-(P>0 \cdot 25)$ and 10-d-old $(P>0 \cdot 5)$ piglets (Table 1$)$.

\section{DISCUSSION}

Several methods have been used to obtain blood samples from sucking piglets. These methods include slaughter and exsanguination of the piglet (Lodge et al. 1978), the insertion of indwelling arterial and venus cannulas (Bengtsson et al. 1969; Flecknell et al. 1988), puncture of the orbital sinus (Friend \& Brown, 1971) and blind puncture of the anterior vena cava (Bengtsson et al. 1969; Seerely \& Poole, 1974). While these methods were not appropriate for routine use in piglets housed in a commercial piggery, Holmes et al. (1990) have shown that repeated blood samples can be obtained from the ear veins of piglets. Indeed, this method, which is relatively non-invasive, was successfully applied in the current study to obtain frequent small volumes of blood $(60 \mu \mathrm{l})$ from piglets.

During times of moderate stress, activation of the sympathetic nervous system causes an increase in both adrenaline and noradrenaline, which contribute to a change in the metabolic status of the animal (Himms-Hagen, 1967; Hingerty \& O'Boyle, 1972). One of the effects of these hormones is to elevate the concentration of glucose in the blood (Himms-Hagen, 1967). Indeed, the hyperglycaemia associated with stress was demonstrated during the collection of blood samples from the ear veins of piglets (Holmes et al. 1990). Our study has shown that the administration of an oral dose of water $\left(\mathrm{H}_{2} \mathrm{O}\right.$ treatment $)$ to the 2-d-old piglets produced a similar degree of hyperglycaemia to that associated with the handling and sampling in the ND treatment (Table 1). However, in the 10-d-old piglets the oral dosing procedure used in the $\mathrm{H}_{2} \mathrm{O}$ treatment resulted in an increased hyperglycaemic response above that associated with the handling and sampling alone (ND treatment; Table 1). Indeed, the increase in the concentration of glucose in the plasma of the 10-d-old piglets following the $\mathrm{H}_{2} \mathrm{O}$ treatment was not significantly different from the increase which occurred following the doses of either galactose (GAL treatment) or galactose plus glucose (GAL + GLU treatment).

In contrast to glucose, the concentration of galactose remained constant in the plasma of the 2- and 10-d-old piglets during the ND and $\mathrm{H}_{2} \mathrm{O}$ treatments used in our studies (Fig. 2). These results demonstrated that the concentration of galactose in the plasma after oral dosing was unaffected by the stress associated with the experimental procedures and validate the experimental procedures we have used for the investigation of intestinal absorption and metabolism of galactose.

The half-life of galactose in the plasma of human adults after an intravenous dose of galactose $(350 \mathrm{mg} / \mathrm{kg}$ ) was reported to be $10.9 \mathrm{~min}$ with a range of $5-15 \mathrm{~min}$ (Hjelm \& Sjolin, 1966). Furthermore, the half-life of galactose in human neonates ( $100 \mathrm{~h}$ postpartum) was found to be about $10 \mathrm{~min}$ (Hjelm \& Sjolin, 1966). These findings are consistent with our results which demonstrated that the half-life of galactose in the plasma of piglets following an intravenous dose of galactose $(60 \mathrm{mg} / \mathrm{kg}$ ) was $7.98(\mathrm{SE} 0.28)$ min and $9 \cdot 10$ (SE 1.29) min for the 2- and 10-d-old piglets respectively. Following oral doses of galactose 
the half-life of galactose was estimated from the postabsorption decrease in the concentration of galactose in the plasma (Fig. 2). These half-lives of 9.29 (SE 2.97) min and $7 \cdot 15$ (SE $2 \cdot 25$ ) $\mathrm{min}$ for the 2 - and 10 -d-old piglets respectively were not significantly different to the half-lives calculated for the intravenous injections of galactose. Thus, given sufficient time for intestinal absorption of galactose and the distribution in the circulation and extracellular water, the route of galactose administration did not affect the values obtained for estimation of the half-life of galactose in the blood.

The elimination rates for galactose (determined from intravenous injections) for human infants has been reported to range from $7-10 \% / \mathrm{min}$ (Kliegman \& Sparks, 1985) to $2 \cdot 3-6.9 \% / \mathrm{min}$ (Siegal et al. 1988). Using a scaled reciprocal $\left(0.693 / t^{\frac{1}{2}} \times 100\right)$ to calculate the elimination rate (Pribylova et al. 1979), we found similar rapid elimination rates for galactose from the plasma of 2 - and 10 -d-old piglets $(8.68 \% / \mathrm{min}$ and $7.61 \% / \mathrm{min}$ respectively). In several species of neonatal animals galactose is incorporated into hepatic glycogen more rapidly than glucose and the activity of galactokinase $(E C 2.7 .1 .6)$ in the liver is greater than that of either hexokinase $(E C 2.7 .1 .1)$ or glucokinase $(E C 2.7 .1 .2)$ (Kliegman \& Sparks, 1985). Furthermore, Katz et al. (1986) reported that, after the administration of an oral dose of glucose to rats, much of the absorbed glucose passed through the liver and was metabolized by the peripheral tissues. These findings suggest that, while most of the absorbed galactose is taken up by the liver and is available to replenish hepatic glycogen, most of the glucose passes through the liver without being metabolized and is available to correct hypoglycaemia and serve as an obligatory energy supply to tissues such as the brain. In this context the galactose moiety of lactose may be important for metabolic homeostasis of young mammals. Indeed, newborn piglets have very little insulation and low fat reserves (10-20 g/ kg body weight; Mellor \& Cockburn, 1986) and, therefore, the galactose moiety of lactose would appear to facilitate optimally the replenishment of hepatic glycogen for postprandial glucose homeostasis.

The turnover of galactose in piglets under basal conditions was calculated from its halflife $(9 \cdot 1 \mathrm{~min})$, basal concentration in blood (17.8 (SE 4.1) $\mu \mathrm{M}$, mean for the $\mathrm{ND}$ and $\mathrm{H}_{2} \mathrm{O}$ treatments; Fig. 2) and an estimate of the volume of extracellular fluid ( $500 \mathrm{~g} / \mathrm{kg}$ body weight; Flynn et al. 1968). The basal turnover rate for galactose in the piglets was $0.1 \mathrm{mg} / \mathrm{min}$ per $\mathrm{kg}$ body weight which is considerably lower than the turnover rate for galactose in lambs (1.4 mg/min per kg body weight; Kaempf et al. 1990). Furthermore, the basal turnover rate for galactose in the piglet was about 80 -fold less than that for glucose $(8 \cdot 1 \mathrm{mg} / \mathrm{min}$ per $\mathrm{kg}$ body weight in piglets; Flecknell et al. 1988). Nevertheless, the basal turnover rate for galactose is metabolically significant and the endogenous source of galactose required to maintain its basal concentration in blood remains unidentified.

When glucose was included with a galactose dose there was a reduction in the maximum concentration of galactose in the blood in man and guinea-pigs, no difference in the rat and an increased response in mice (Newstead, 1979; Williams \& Owens, 1984). In comparison, we demonstrated that the presence of glucose did not lower the galactose response in the peripheral blood of piglets (Fig. 2, Table 1). This indicates that the effect of glucose on the metabolism of galactose in piglets was similar to rats and unlike that of man, guinea-pigs or mice.

The authors would like to thank Sandra Pember and Melinda Hartmann for their excellent technical assistance, Wandalup Farms for the availability of the piglets and their facilities and acknowledge the Pig Research and Development Corporation for granting both a Junior Research Fellowship and the financial support for this project. 


\section{REFERENCES}

Arthur, P. G., Kent, J. C. \& Hartmann, P. E. (1989). Microanalysis of the metabolic intermediate of lactose synthesis in human milk and plasma using bioluminescent methods. Analytical Biochemistry 176, 449-456.

Barber, R. S., Braude, R. \& Mitchell, K. G. (1955). Studies on milk production of large white pigs. Journal of Agricultural Science 46, 97-118.

Bengtsson, G., Gentz, J., Hakkarainen, J., Hellström, R. \& Persson, B. (1969). Plasma levels of FFA, glycerol, $\beta$-hydroxybutyrate and blood glucose during the postnatal development of the pig. Journal of Nutrition 97 , $311-315$.

Bergmeyer, H. U. \& Bern't, E. (1974). D-glucose: Determination with glucose oxidase and peroxidase. In Methods of Enzymatic Analysis, 2nd ed., vol. 3, pp. 1205-1215 [H. U. Bergmeyer, editor]. New York: Academic Press.

Engelhardt, W. (1966). Swine cardiovascular physiology. In Swine in Biomedical Research, pp. 307-329 [L. K Bustad and R. O. McClellan, editors]. Washington, USA: Battelle Memorial Institute.

Flecknell, P. A., Wootton, R., Royston, P. \& John, M. (1988). Glucose homeostasis in the newborn. Effects of oral feeding on response to fasting and intravenous glucose infusion in neonatal piglets. Biology of the Neonate 54, 356-362.

Flynn, M. A., Hanna, F., Long, C. H., Asfour, R. Y., Lutz, R. N. \& Zobrisky, S. E. (1968). Deuterium-oxide dilution as a predictor of body composition in children and pigs. In Body Composition in Animals and Man, pp. 480-491. Washington D.C.: National Academy of Sciences.

Friend, D. W. \& Brown, R. G. (1971). Blood sampling from suckling piglets. Canadian Journal of Animal Science 51, 547-549.

Himms-Hagen, J. (1967). Sympathetic regulation of metabolism. Pharmacological Reviews 19, 367-461.

Hingerty, D. \& O'Boyle, A. (1972). Clinical Chemistry of the Adrenal Medulla. Illinois, USA: Charles C. Thomas.

Hjelm, M. \& Sjolin, S. (1966). Changes in the elimination rate from blood of intravenously injected galactose during the neonatal period. Scandinavian Journal of Clinical and Laboratory Investigation 18, 126-131.

Holmes, M. A., Arthur, P. G. \& Hartmann, P. E. (1990). Changes in the concentrations of glucose and galactose in the peripheral blood of sucking piglets. Journal of Dairy Research 57, 331-337.

Kaempf, J. W., Battaglia, F. C.\& Sparks, J. W. (1990). Galactose clearance and carbohydrate metabolism across the gastrointestinal tract in the newborn lamb. Metabolism 39, 698-703.

Katz, J., Kuwajima, M., Foster, D. W. \& McGarry, J. D. (1986). The glucose paradox: new perspectives on hepatic carbohydrate metabolism. Trends in Biological Sciences 3, 135-140.

Kliegman, R. M. \& Sparks, J. W. (1985). Perinatal galactose metabolism. Journal of Pediatrics 107, 831-841.

Lodge, G. A., Sarkar, N. K. \& Kramer, J. K. G. (1978). Fat deposition and fatty acid composition in the neonatal pig. Journal of Animal Science 47, 497-504.

Mellor, D. J. \& Cockburn, F. (1986). A comparison of energy metabolism in the newborn infant, piglet and lamb. Quarterly Journal of Experimental Physiology 71, 361-379.

Mount, L. E. \& Ingram, D. L. (1971). The Pig as a Laboratory Animal. London: Academic Press.

Newstead, G. C. (1979). Serum carbohydrate levels following galactose and galactose plus glucose given to rats. Proceedings of the Nutrition Society 38, 38A.

Pribylova, H., Sternova, H. \& Kozlova, J. (1979). Plasma insulin, carbohydrate and free fatty acid changes in newly born infants of diabetic and non-diabetic mothers after loading with glucose, fructose and galactose. Physiologia Bohemoslovaca 36, 193-197.

Ramirez, C. G., Miller, E. R., Ullrey, D. E. \& Hoefer, J. A. (1963). Swine hematology from birth to maturity. 3. Blood volume of the nursing pig. Journal of Animal Science 22, 1068-1074.

Seerley, R. W. \& Poole, D. R. (1974). Effect of prolonged fasting on carcass composition and blood fatty acids and glucose of neonatal swine. Journal of Nutrition 104, 210-217.

Siegal, C. D., Sparks, J. W. \& Battaglia, F. C. (1988). Patterns of serum glucose and galactose concentrations in term newborn infants after milk feeding. Biology of the Neonate 54, 301-306.

Tygstrup, N. \& Winkler, K. (1954). Kinetics of galactose elimination. Acta Physiologica Scandinavica 32, $354-362$.

Williams, C. A. \& Owens, A. M. (1984). The influence of glucose on the plasma galactose response to galactose in the rat, guinea-pig and mouse. Proceedings of the Nutrition Society 43, 58A.

Williams, C. A., Philips, T. \& Macdonald, I. (1983). The influence of glucose on serum galactose levels in man. Metabolism 32, 250-256.

Yeh, K. C. \& Kwan, K. C. (1978). A comparison of numerical integrating algorithms by trapezoidal, lagrange and spline approximation. Journal of Pharmacokinetics and Biopharmaceutics 6, 79-98. 